\title{
SDC1 Gene
}

National Cancer Institute

\section{Source}

National Cancer Institute. SDC1 Gene. NCI Thesaurus. Code C96912.

This gene is involved in cell adhesion, signal transduction and cytoskeleton org anization. 